Special issue of the 2nd International Conference on Computational and Experimental Science and Engineering (ICCESEN 2015)

\title{
The Method and Software for the Solution of Dynamic Waves Propagation Problem in Elastic Medium
}

\author{
Z. Akhmetova*, S. Zhuzbaev and S. Boranbayev \\ L.N. Gumilyov Eurasian National University/Department of Information Systems, Astana, Kazakhstan

\begin{abstract}
This paper describes the numerical method for the solution to the problem of propagation of dynamic waves in elastic media - the bicharacteristics method with the usage of the ideas of the splitting method. The bicharacteristics method is one of the most convenient methods for software creating. In this research paper we have worked on the solution for non-stationary problem of the homogeneous isotropic elastic body dynamics using the bicharacteristics method, based on which the "ProgWave" software was designed. With this software, we have obtained the plots of isolines of normal and tangent tensions, which are very important for the studies of non-stationary dynamic waves propagation in flat elastic bodies, in engineering practice at construction designs calculation, in problems of mechanical engineering, etc.
\end{abstract}

DOI: 10.12693/APhysPolA.130.352

PACS/topics: $89.20 . \mathrm{Ff}$

\section{Introduction}

The need for qualitative and quantitative analysis of the dynamic effects of the stress-strained state is increasing because of the connection with the various fields of engineering, structural elements, working in the dynamic load conditions. As a result of the dynamic loads, an elastic wave takes place in the testing body and a reliable calculation of it helps in evaluating the strength and reliability of the entire design and technology.

Nowadays the numerical methods of spatial characteristics [1-3], finite elements [4], the boundary integral equations [5], etc., are used for solving dynamic problems in elastic media.

In this paper, we offer the bicharacteristics method with the usage of ideas of splitting method for solution of the homogeneous isotropic elastic body [3, 6-9]. The advantage of this method is that it allows approaching the maximum dependence domain of the final and differential equation to the dependence area of the initial differential equation [10-12].

Many software programs are based on numerical methods as well as methods of finite elements and boundary elements. The originality of this research work is in the design of the software, based on bicharacteristics method, which is one of the most convenient methods for design applications.

\section{Statement of problem}

Let us consider an elastic semi-strip of final width, which in the Cartesian system of $x_{1} O x_{2}$ coordinates occupies the area $0<x_{2}<\infty,\left|x_{1}\right|<l$ (Fig. 1). At an initial timepoint the body is in the state of rest:

$$
\vartheta_{i}=0, \quad \sigma_{i j}=0, \quad(i, j=1,2) .
$$

\footnotetext{
*corresponding author; e-mail: zaigura@mail.ru
}

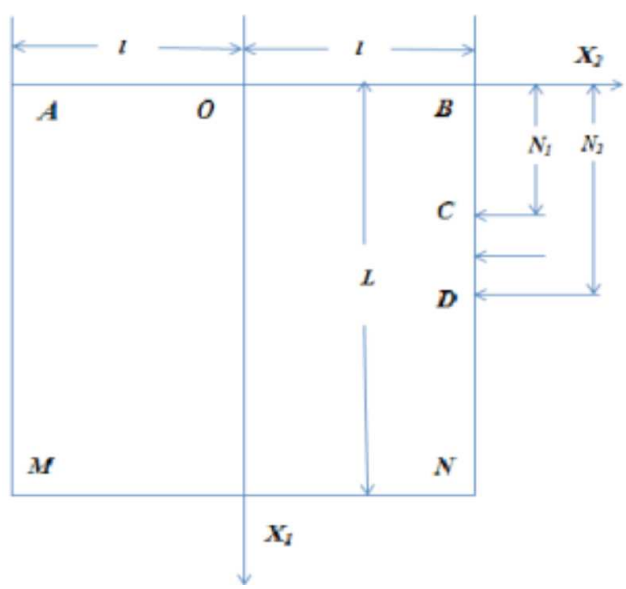

Fig. 1. The area of study.

At any other time on the site $N_{1} \leq x_{2} \leq N_{2}, x_{1}=l$, on the border $\mathrm{BN}$ acts an uniformly distributed normal load transient $f(t)$, which varies according to sine law

$$
\begin{aligned}
\sigma_{22}(t) & =\left\{\begin{array}{l}
-A \sin (\omega t), \quad 0 \leq t \leq S_{1}, \\
0, t \geq S_{1},
\end{array}\right. \\
\sigma_{21}(t) & =0
\end{aligned}
$$

Where $S_{1}$ is action time of the load and $\omega=\pi / S_{1}$. Other part of border of a semi-strip is free from any influence:

$$
\begin{gathered}
\sigma_{11}(t)=0, \sigma_{12}(t)=0, x_{1}=0,\left|x_{1}\right| \geq l, \\
\sigma_{22}(t)=0, \sigma_{21}(t)=0, \\
0 \leq x_{1} \notin\left(N_{1}, N_{2}\right),\left|x_{2}\right|=l .
\end{gathered}
$$

Under existing conditions it is necessary to investigate an elastic body tension at $t>0$.

\section{The defining equations of bicharacteristics}

In order to solve the problem with given initial and boundary conditions, the system of the equations consisting of the movement and ratios equations of the generalized Hooke's law is used: 


$$
\sigma_{i \beta, \beta}=\rho \frac{\partial^{2} u_{i}}{\partial t^{2}}
$$

and

$$
\sigma_{i j}=\lambda u_{\beta, \beta} \delta_{i j}+\mu\left(u_{i, j}+u_{i, j}\right)
$$

where $\rho$ is density, $\lambda, \mu$ are Lama's constants, $\delta_{i j}$ is Kronecker delta.

For convenience independent dimensionless variables and required sizes are defined [3].

$$
\begin{gathered}
\bar{t}=\frac{t c_{1}}{b}, \bar{x}_{i}=\frac{x_{i}}{b}, \bar{v}_{i}=\frac{1}{c_{1}} \frac{\partial u_{i}}{\partial t}, \bar{\sigma}_{i j}=\frac{\sigma_{i j}}{\rho c_{1}^{2}}, \\
\gamma_{12}=\frac{c_{2}}{c_{1}}, \gamma_{11}=1-2 \gamma_{21}^{2}, \quad(i, j=1,2),
\end{gathered}
$$

where $b$ is reference length, $c_{1}=\sqrt{\frac{\lambda+2 \mu}{\rho}}, c_{2}=\sqrt{\frac{\mu}{\rho}}$ are datum speeds.

After the integration of non-dimensional variables, the motion equations (4) and differentiated over time correlation of the generalized Hooke's law (5) take the form:

$$
\left\{\begin{array}{l}
\dot{\vartheta}_{1}=\sigma_{11,1}+\sigma_{12,2}, \\
\dot{\vartheta}_{1}=\sigma_{21,1}+\sigma_{22,2}, \\
\dot{\sigma}_{11}=\vartheta_{1,1}+\gamma_{11} \sigma_{2,2}, \\
\dot{\sigma}_{22}=\gamma_{11} \vartheta_{1,1}+\sigma_{2,2}, \\
\dot{\sigma}_{12}=\gamma_{12}^{2}\left(\vartheta_{1,2}+\vartheta_{2,1}\right) .
\end{array}\right.
$$

For equations of bicharacteristics and conditions on them, let us split the two-dimensional system (7) into a one-dimensional, by applying ideas of K.A. Bagrinovski and S.K. Godunov of splitting multidimensional $t$-hyperbolic systems into one-dimensional systems on $x_{k}=$ const [9]

$$
\left\{\begin{array}{l}
\dot{v}_{i}-\sigma_{i j, j}=a_{i j}, \\
\dot{\sigma}_{i j}-\lambda_{i j}^{2} v_{i, j}=b_{i j},
\end{array}\right.
$$

where $a_{i j}=\sigma_{i k, k} ; \quad \lambda_{i j}=\sigma_{i j}+\gamma_{12}\left(1-\delta_{i j}\right) ; b_{i j}=$ $\left[\gamma_{11} \delta_{i j}+\gamma_{12}^{2}\left(1-\delta_{i j}\right)\right] \vartheta_{p, k}$

Hence below $i, j, k, p=1,2 ; p \neq i, k \neq j$. From here, using known methods of obtaining the differential equations bicharacteristics and conditions on them, we get:

$$
\mathrm{d} x_{j}= \pm \lambda_{i j} \mathrm{~d} t, \quad \mathrm{~d} \sigma_{i j} \pm \lambda_{i j} \mathrm{~d} v_{i}=\left(b_{i j} \pm \lambda_{i j} a_{i j}\right) \mathrm{d} t .(9)
$$

This body is divided into square cells, with sides of $\Delta x_{1}=\Delta x_{2}=h$. The double points are searched function values $\vartheta_{i}, \sigma_{i j}$ at various time points with step $\tau$. The grid of dots, on the basis of which the difference scheme is built, other than these mentioned double points, contains points formed by the intersection with bicharacteristics hyper planes $t=$ const. Accepted pattern consists of $O$ node and $E_{i j}^{ \pm}$points, separated from the point $O$ by distance $\lambda_{i j} \tau$. In the future discussion values of the functions at the point $O$ are denoted by upper index " 0 ", values at the points $E_{i j}^{ \pm}$are denoted by lower index " $i \jmath$ " and the upper index " \pm " (for example $\sigma_{i j}^{ \pm}$), and values at point $A$ are not not denoted by an additional index [13-16].

The integration of Eqs. (7) from the point $O$ to the point $A$ and the relations (9) from the point $E_{i j}^{ \pm}$ to point $A$ using trapezoid method allows to obtain the expression of the following form

$$
\begin{gathered}
\vartheta_{i}=\vartheta_{i}^{0}+\frac{\tau}{2}\left(\sigma_{i j, j}+a_{i j}+\vartheta_{i}^{0}\right), \\
\dot{\sigma}_{i j}=\sigma_{i j}^{0}+\frac{\tau}{2}\left(\lambda_{i j}^{2} v_{i, j}+b_{i j}+\dot{\sigma}_{i j}^{0}\right), \\
\sigma_{i j}-\sigma_{i j}^{ \pm} \mp \lambda_{i j}\left(v_{i}+v_{i}^{ \pm}\right)= \\
\frac{\tau}{2}\left(b_{i j}+b_{i j}^{ \pm} \mp \lambda_{i j}\left[a_{i j}+a_{i j}^{ \pm}\right]\right) .
\end{gathered}
$$

By summing up and subtracting system equations with identical indexes pairs, we get

$$
\begin{aligned}
& v_{i, j}=v_{i, j}^{0}+\tau\left(\sigma_{i j, j j}^{0}+a_{i j, j}^{0}\right), \\
& \sigma_{i j, j}=\sigma_{i j, j}^{0}+\tau\left(\lambda_{i j}^{2} v_{i, j j}^{0}+b_{i j, j}^{0}\right) .
\end{aligned}
$$

\section{Final remarks}

To solve this problem we have developed the "ProgWave" software, which makes it possible to obtain the plots of isolines of normal and tangent tensions.

"ProgWave" is based on bicharacteristics method with the usage of ideas of splitting method. The decision algorithm is realized in the algorithmic language on the grid with a step of $\tau=0.025, h=\Delta x_{1}=\Delta x_{2}=0.05$. In this problem statement the calculation was made for steel $\rho=7900 \mathrm{~kg} / \mathrm{m}^{3}, c_{1}=5817 \mathrm{~m} / \mathrm{s}, c_{2}=3109 \mathrm{~m} / \mathrm{s}$, for the following values of basic parameters: $A=0.5$, $\omega=4.5, l=5 h, L=70 h, N_{1}=10 h, N_{2}=14 h$.

The "ProgWave" software is universal and it is possible to change the function of the non-stationary load and parameters of the design material depending on the statement of problem. In this paper using the software "ProgWave" we have obtained isolines of normal and tangent tensions at several moments of time.

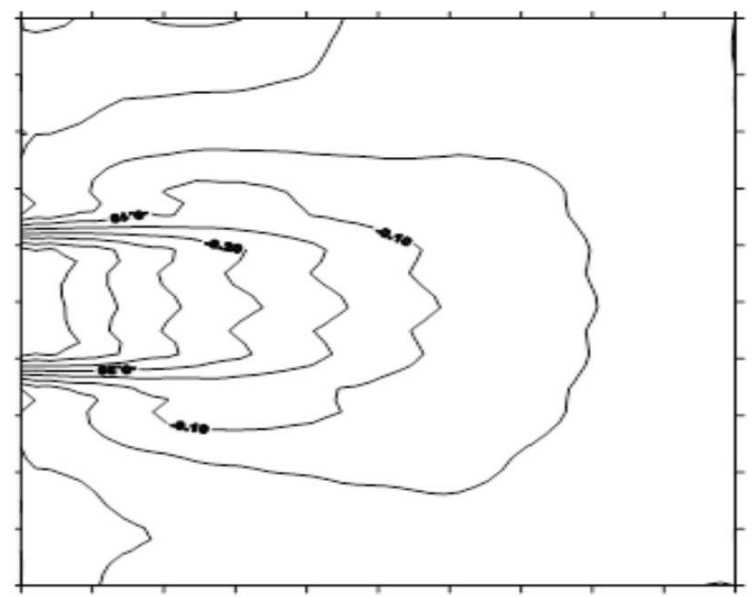

Fig. 2. Isolines of normal tension.

In Figs. 2 and 3 one can see the isolines of normal $\sigma_{11}$ and tangent $\sigma_{12}$ tension, corresponding to a time point $t=20 \tau$. During this time, the boundary perturbation propagating from the influenced local site has traveled a distance of $10 \mathrm{~h}$ and has reached the opposite border. 


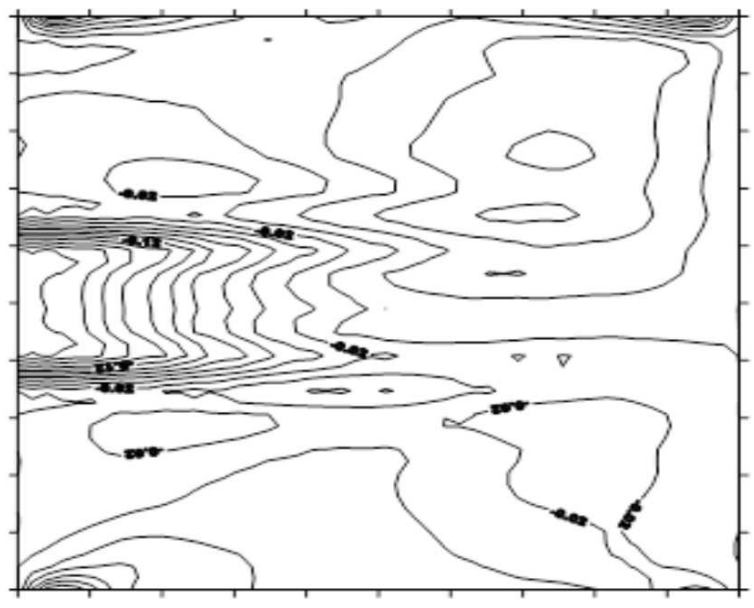

Fig. 3. Isolines of tangent tension.

At a timepoint $t=40 \tau$, the tension fields symmetry, characteristic for $t=20 \tau$, with respect to axis at $x_{1}=12 h$ is still visible in the vicinity of the symmetry axis. With the increasing separation from this axis, the symmetry of isolines becomes broken. This result is explained by the influence on the nature of distribution of tension from a free end $\mathrm{AB}$ in the field of $x_{1} \leq N_{1}$ and absence of similar effects in the field of $x_{1} \leq N_{2}$.

\section{Conclusions}

In the design and construction of engineering structures for more economical use of materials it is necessary to consider not only the static load on structures, but also the dynamic one, which may occur, for example, in areas with high seismic activity. Powerful dynamic load cannot be determined without a full study of the space-time picture of the state of stress of the solid object, appearing during the propagation of elastic waves.

In this paper, the solution of problem of nonstationary dynamic waves propagation in flat elastic bodies was offered using bicharacteristics numerical method. The practical implementation has been carried out by creating "ProgWave" software.

The obtained results and software can be used in research of non-stationary dynamic waves propagation in flat elastic bodies, in engineering practice at construction designs calculations, in problems of mechanical engineering, etc.

\section{References}

[1] R.D. Clifton, Mechanics. Moscow 1, 103 (1968).

[2] V.V. Reker, Appl. Mech. E 1, 121 (1970).

[3] T.D. Karimbayev, S.S. Dzhuzbayev, in: Tez. Dokl. Mezhd. Konf., Kiev 1992, p. 18.

[4] Sh.M. Aytaliyev, Zh.K. Masanov, I.B. Baymakhanov, N.M. Makhmetova, Numerical methods of the solution of problems of mechanics of a deformable solid body, Baspa, Karaganda 1987, p. 37.

[5] Sh.M. Aytaliyev, L.A. Alekseeva, Sh.A. Dildabayev, N.B. Zhanbyrbayev, Metod of the boundary integrated equations in problems of dynamics elastic many connected deformable solid bodyies ph, Aimaty, Alma-Ata 1992, p. 131.

[6] G.T. Tarabrin, Mechanics Calc. Construct. 4, 38 (1981).

[7] G.T. Tarabrin, Metallurgical Sci. 3, 193 (1979).

[8] G.T. Tarabrin, Construction mechanics and calculation of constructions, Moscow 1980, p. 53.

[9] B.T. Bayteliyev, S.S. Dzhuzbayev, in: Tez. Dokl. Vsesoyuzn. Simpoziuma "Arheology of Soil", RusPolifraf, Volgograd 1985, p. 47.

[10] Y. Ru, G.F. Wang, T.J. Wang, J. Vib. Acoust. 131, 061011 (2009).

[11] S.K. Kanaun, V.M. Levin, Int. J. Solids Struct. bf 79, 260 (2005).

[12] V.K. Kinra, M.S. Petraitis, S.K. Datta, Int. J. Solids Struct. 16, 301 (1980).

[13] R.B. Yang, J. Appl. Mech. bf 31, 78 (2003).

[14] J.-Y. Kim, Int. J. Solids Struct. bf 124, 201 (2004).

[15] S.K. Kanaun, V.M. Levin, F.J. Sabina, Wave Motion 40, 69 (2004).

[16] L.Ya. Cosachevskiy, Prikl. Mat. Mekh. 23, 1115 (1959). 